ISSN 0258-7122 (Print), 2408-8293 (Online)

Bangladesh J. Agril. Res. 41(2): 273-286, June 2016

\title{
GENETIC DIVERSITY OF MUSKMELON USING MULTIVARIATE TECHNIQUE
}

\author{
S. RAHMAN ${ }^{1}$, M. A. K. MIAH ${ }^{2}$ AND H. RAHMAN ${ }^{3}$
}

\begin{abstract}
An experiment was conducted at the experimental farm of Plant Genetic Resources Centre (PGRC), Bangladesh Agricultural Research Institute (BARI), Gazipur in 2011 to estimate genetic diversity through multivariate technique. Based on multivariate analysis and application of covariance matrix for nonhierarchical clustering, 64 genotypes of muskmelon were grouped into six clusters to indicate the existence of considerable diversity among the genotypes. The cluster IV was consisted of single genotypes (BD2303). The highest number of genotypes possessed in Cluster I. The first principal axis largely accounted for the variation among the genotypes which alone contributed $25.65 \%$ of the variations. The highest inter genotypic distance (2.878) was observed between the genotypes BD2303 and BD2313 followed by the genotypes BD2303 and BD2314 (2.808). The highest intra cluster distance was computed for cluster III (0.839) followed by cluster I (0.751). Cluster VI showed the least intra cluster distance which indicated that the genotypes in this cluster were more or less homogeneous. The inter cluster distances were larger than the intra cluster distances suggesting wider genetic diversity among the genotypes of different clusters. Cluster mean pointed out the heavier fruit in cluster IV $(2533.3 \mathrm{~g})$. The size of this cluster was also far different from all other clusters. Similarly, the highest total fruit weight per plant was found in cluster IV $(13.5 \mathrm{~kg})$ which was also far different from other clusters. So it revealed that genotypes of this cluster could be used for developing high yielding variety. Cluster VI showed the highest brix reading $(5.6 \%)$. Therefore, the genotypes of this cluster could be used for the development of sweet muskmelon variety. Hybridization between the genotypes of cluster IV and those of cluster VI could develop high yielding sweet muskmelon variety(s).
\end{abstract}

Keywords: Genetic diversity, Cucumis melo, Cluster and Multivariate analysis.

\section{Introduction}

Nature of out crossing in muskmelon is always generating genetic diversityin this crop (Saxena, 2005 and Hancock, 2012). Moreover, genetic diversity is essential for a plant breeding programme. A new variety as per farmer's demand can be developed from an assembled diverse genetic stock of any crop.

${ }^{1}$ Senior Scientific Officer, Plant Genetic Resources Centre (PGRC), Bangladesh Agricultural Research Institute (BARI), Gazipur, ${ }^{2}$ Professor, Department of Genetics and plant breeding, Bangabandhu Sheikh Mujibur Rahman Agricultural University (BSMRAU), Gazipur, ${ }^{3}$ Assistant professor, Department of Entomology, BSMRAU, Gazipur, Bangladesh. 
So success of any breeding program depends much on the genetic diversity available and the judicious selection of parents. The importance of genetically diverse genotypes as a source of obtaining transgressive segregants with desirable combinations has been reported by several workers (Baenziger et al., 2006 and Stuthman et al., 2007). The importance of genetic diversity in any crop improvement programme has been stressed both in self and cross pollinated crops (Flowers, 2004).

Genetic resources are, in the sense, the building blocks and also fundamental not only to a crop improvement program, but also for the very survival of the species in time and space (Swaminathan, 1983). Moreover, evaluation of genetic diversity is important to know the source of genes for particular trait within the available germplasm (Tomoka, 1991). Multivariate analysis by means of Mahalanobis' $\mathrm{D}^{2}$ statistics is a useful tool in quantifying the degree of genotypic divergence among biological populations and to assess the relative contribution of different components to the total divergence both at inter and intra-cluster levels (Murty and Arunachalam, 1966; Das and Gupta, 1984). From the plant breeding point of view the degree of genetic diversity between two parents is an index for determining the hybridity over parents or nature of the segregants in the follow-up generation. $\mathrm{D}^{2}$ statistics can help in selecting desirable parents for achieving desired goal by the breeder. Though information on genetic divergence is available in most of the crops, such information in muskmelon is very rare (Kalloo et al., 1982). Considering the above facts, the present investigation was undertaken to assess the genetic diversity among the collected germplasm and to identify the diverse parents for use in further genetic study.

\section{Materials and Method}

The experiment was conducted at the experimental farm of Plant Genetic Resources Centre (PGRC), Bangladesh Agricultural Research Institute (BARI), Gazipur in 2011. The data on quantitative parameter of muskmelon was recorded and used to estimate genetic diversity through multivariate technique. The quantitative parameters were as follows:
a. Fruit length $(\mathrm{cm})$
b. Fruit width $(\mathrm{cm})$
c. Fruit girth $(\mathrm{cm})$
d. Flesh thickness $(\mathrm{cm})$
e. Cavity diameter $(\mathrm{cm})$
f. Fruit size (g)
g. Number of fruits per plant
h. Number of seeds per fruit
i. 100 seed weight $(\mathrm{g})$
j. Seed yield per plant (g)
k. Total fruit weight $(\mathrm{kg})$
1. Brix \% 


\section{Analysis of data}

Mean data for each character was subjected to multivariate analysis techniques viz. Principal component analysis (PCA), Principal coordinate analysis (PCO), Canonical vector analysis (CVA) and Cluster analysis (CLSA) using GENSTAT 5.13 software.

\section{Principal component analysis (PCA)}

Principal component analysis is one of the multivariate techniques to know the interrelationships among several characters and can be done from the sum of squares and product matrix for the characters. Principal components were computed from the correlation matrix and genotypic scores obtained for the first component and succeeding components with latent roots greater than unity (Jeger et al., 1983). The latent roots are called "Eigen values". The first component has the property of accounting for maximum variance. The PCA displays most of the original variability in a smaller number of dimensions, since it finds linear combinations of a set of variate that maximize the variation contained within them. Contributions of the different characters towards divergence are discussed from the latent vectors of the two principal components.

\section{Principal coordinate analysis (PCO)}

Principal coordinate analysis is equivalent to PCA but it is used to calculate interunit distances. Through the use of all dimensions of $p$ it gives the minimum distances between each pair of the $\mathrm{N}$ points using similarity matrix (Digby et $a l ., 1989)$. Inter-distances between genotypes were studied by PCO.

\section{Canonical variate analysis (CVA)}

CVA complementary to $\mathrm{D}^{2}$-statistic is a sort of multivariate analysis where canonical vectors and roots representing different axes of differentiation and the amount of variation accounted for by each of such axes, respectively are derived. Canonical variate analysis finds linear combination of original variability that maximizes the ratio of between groups to within groups variation, thereby giving functions of the original variables that can be used to discriminate between the groups. Thus in this analysis, a series of orthogonal transformations sequentially maximize the ratio of among groups to within group variations.

\section{Cluster analysis (CLSA)}

Genotypes were divided into groups on the basis of a data set into some number of mutually exclusive groups. The clustering was done using nonhierarchical classification. In Genstat, the algorithm is used to search for optimal values of the chosen criterion. The optimal values of the criteria followed by some initial 
classification of the genotypes into required number of groups, the algorithm repeatedly transfers genotypes from one group to another so long as such transfer improved the value of the criterion. No further transfer can be found to improve the criterion. The algorithm switches to second stages that examine the effect swapping two genotypes of different classes, and so on.

\section{Computation of average Intra-cluster distance}

Computation of Average Intra-Cluster distance for each cluster was calculated by taking possible $\mathrm{D}^{2}$ values within the members of a cluster obtained from the PCO after the clusters are formed. The formula utilized was $\sum D^{2} / n$, where $\sum D^{2}$ is the sum of distances between all possible combinations (n) of the genotypes included in a cluster. The square root of the average $\mathrm{D}^{2}$ values represents the distance (D) within cluster.

\section{Cluster diagram}

It was drawn using the values between and within cluster distances, which presents a momentary idea of the pattern of diversity among the genotypes included in a cluster.

\section{Computation of average inter - cluster distances}

The procedure for calculating inter-cluster distance between cluster II and I, between cluster III and I, between cluster IV and I, between cluster V and I, between cluster VI and I and between cluster II and III and so on. The clusters were taken one by one and their distances from other clusters were calculated.

\section{Selection of varieties for future hybridization programme}

Divergence analysis is usually performed to identify the diverse genotypes for hybridization programme. The genotypes grouped together are less divergent among themselves than different clusters. Clusters separated by the largest statistical distance $\left(\mathrm{D}^{2}\right)$, express the maximum divergence among the genotypes included into these different clusters.

\section{Results and Discussion}

\section{Principal component analysis}

Eigen values of twelve principal component axes and percentage of total variation accounting for them obtained from the principal component analysis are presented in Table 1 . The result revealed that the first principal axis largely accounted for the variation among the genotypes which alone contributed $25.25 \%$ of the variations. While the first six Eigen values for the principal component axes of genotypes accounted for $85.38 \%$ of the total variation among 
12 characters describing 64 genotypes while the former three accounted for $56.6 \%$. Henane et al. (2013) reported that the first three axes explained $89.86 \%$ of the observed phenotypic diversity and the first principal component explained $54.5 \%$ of the total variance.

Table 1. Eigen values and percentage of variation for corresponding 12 component characters in muskmelon genotypes

\begin{tabular}{l|c|c|c}
\hline Principle of component axis & Eigen values & $\begin{array}{c}\text { Percentage of total } \\
\text { values accounted }\end{array}$ & $\begin{array}{c}\text { Cumulative } \\
\text { percentage }\end{array}$ \\
\hline Fruit length (cm) & 3.078 & 25.65 & 25.65 \\
Fruit width (cm) & 1.8974 & 15.81 & 41.46 \\
Fruit girth (cm) & 1.8165 & 15.14 & 56.6 \\
Flesh thickness(cm) & 1.4319 & 11.93 & 68.53 \\
Cavity diameter (cm) & 1.1044 & 9.2 & 77.73 \\
Fruit size (g) & 0.9175 & 7.65 & 85.38 \\
No. of fruits / plant & 0.7087 & 5.91 & 91.29 \\
No. of seeds / fruit & 0.633 & 5.27 & 96.56 \\
100 seed weight (g) & 0.4 & 3.33 & 99.89 \\
Seed yield / plant (g) & 0.0098 & 0.08 & 99.97 \\
Total fruit weight/plant (kg) & 0.0027 & 0.02 & 99.99 \\
Brix \% & 0 & 0 & 99.99 \\
\hline
\end{tabular}

\section{Construction of scatter diagram}

Based on these values of principal component scores 2 and 1 obtained from the principal component analysis, a two dimensional scatter diagram $\left(\mathrm{Z}_{1} \mathrm{Z}_{2}\right)$ using component scores 1 as $\mathrm{X}$ axis and component scores 2 as $\mathrm{Y}$ axis was constructed which has been presented in Fig.1. The positions of the genotypes in the scatter diagram were apparently distributed into 6 groups which indicated the existence of considerable diversity among the genotypes. Significant genetic diversity in muskmelon was also investigated by Escribano and Lazaro (2009); Nasrabadi et al. (2012) and Trimech et al. (2013) using different morphological characters.

\section{Principal coordinate analysis(PCO)}

PCO was done to get inter genotypic distances. The highest inter genotypic distance (2.878) was observed between the genotypes BD2303 and BD2313 followed by the genotypesBD2303 and BD2314 (2.808). The lowest distance (0.249) was observed between genotypes BD2288 and BD2310 followed by BD2259 and BD2309 (0.252). The intra cluster distance wascalculated from inter genotypic distance as suggested by Singh and Chaudhary (1985).The magnitude 
of the intracluster distances were not always proportional to the number of genotypes in the cluster. Zhang et al. (2012) studied on principal of coordinateanalysis in Mediterranean region and south Asian region and found that fruit length and fruit girth dominated in $\mathrm{PC}_{1}$ and $\mathrm{PC}_{2}$, respectively.

The study revealed that the cluster I composed of the largest number of genotypes 20 (Table 2), but intra cluster distance was not necessarily the highest. Statistical distances were representing the index of genetic diversity among the cluster. The highest intra-cluster distance was computed for cluster III $(0.839)$ which consisted of 11 genotypes followed by cluster I (0.751) having 20 genotypes (Table 3). The intra distance of cluster IV is 0.00 since the cluster consisted of only one genotype (BD 2303). Cluster VI showed the least intra cluster distance indicating that the genotypes in this cluster were more or less homogeneous (Fig.2). Henane et al. (2013) observed ahigh degree of polymorphism in muskmelon for almost all of qualitative characters. Moreover, cluster analysis and distribution of populations of PCO separated the varieties in different groups with a divergence variety from the other varieties.

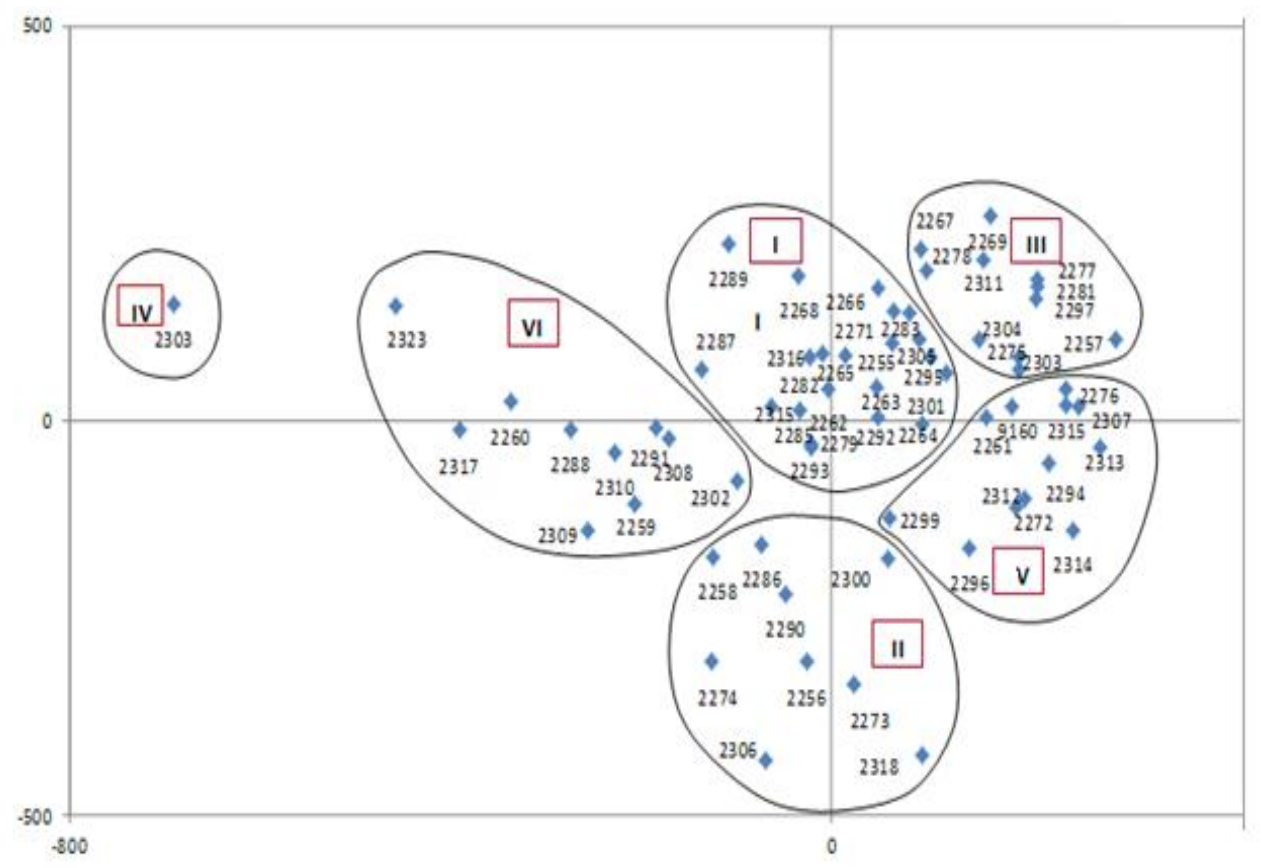

Fig. 1. Scatter diagram of 64 muskmelon genotypes based on their principal component scores super imposed with clustering. 
Table 2. Distribution of 64 genotype in different clusters

\begin{tabular}{|c|c|c|}
\hline Cluster & Genotypes & $\begin{array}{l}\text { Number of } \\
\text { genotypes }\end{array}$ \\
\hline I & $\begin{array}{l}\text { BD-2255, BD-2262, BD-2263, BD-2264, BD-2265, BD-2266, } \\
\text { BD-2268, BD-2271, BD-2279, BD-2282, BD-2283, BD-2285, } \\
\text { BD-2289, BD-2292, BD-2293, BD-2295, BD-2301, BD-2305, } \\
\text { BD-2315, BD-2316 }\end{array}$ & 20 \\
\hline II & $\begin{array}{l}\text { BD-2256, BD-2258, BD-2273, BD-2274, BD-2286, BD-2290, } \\
\text { BD-2300,BD-2306, BD-2318 }\end{array}$ & 9 \\
\hline III & $\begin{array}{l}\text { BD-2257,BD-2267,BD-2269,BD-2275,BD-2277,BD- } \\
\text { 2278,BD-2281,BD-2297,BD-2298,BD-2304,BD-2311 }\end{array}$ & 11 \\
\hline IV & BD-2303 & 1 \\
\hline $\mathrm{V}$ & $\begin{array}{l}\text { BD-2261, BD-2272, BD-2276, BD-2280, BD-2294, BD-2296, } \\
\text { BD-2299,BD-2307, BD-2312, BD-2313, BD-2314, BD-9160 }\end{array}$ & 12 \\
\hline VI & $\begin{array}{l}\text { BD-2259, BD-2260, BD-2287, BD-2288, BD-2291, BD-2302, } \\
\text { BD-2308, BD-2309, BD-2310, BD-2317, BD-2323 }\end{array}$ & 11 \\
\hline
\end{tabular}

\section{Canonical variate analysis}

The CVA was performed to obtain the inter cluster distances (Mahalanobis' $\mathrm{D}^{2}$ Values) The values of inter-cluster distance $\left(D^{2}\right)$ are presented in Table 3. Statistical distances represented the index of genetic diversity among the clusters. The inter cluster distances were larger than the intra-cluster distances suggesting wider genetic diversity among the genotypes of different clusters (Table 4).The inter-cluster distance (Fig. 2) was maximum between clusters III and IV (30.111) followed by clusters IV and V, while the minimum distance was found between clusters I and V (2.934) followed by cluster I and III (3.272). The maximum value of inter-cluster distance indicated that the genotype belonging to this cluster were far diverged. The minimum cluster distance suggesting a close relationship between the genotypes of clusters. These relationships were also reflected in the scatter diagram.

Table 3. Intra and inter cluster distances of 64 muskmelon genotypes

\begin{tabular}{lcccccc}
\hline I & $\mathbf{0 . 7 5 1}$ & & & & & \\
II & 5.271 & $\mathbf{0 . 6 3 6}$ & & & & \\
III & 3.272 & 7.261 & $\mathbf{0 . 8 3 9}$ & & & \\
IV & 27.852 & 26.81 & $\mathbf{3 0 . 1 1 1}$ & $\mathbf{0 . 0 0}$ & & \\
V & 2.934 & 5.196 & 3.106 & 30.107 & $\mathbf{0 . 7 3 0}$ & \\
VI & 4.449 & 4.555 & 6.964 & 23.917 & 6.384 & $\mathbf{0 . 6 3 0}$ \\
& I & II & III & IV & V & VI \\
\hline
\end{tabular}


The genotypes of distant clusters could be used in hybridization programme for obtaining a wide range of variation among the segregate. Similar reports were also made by Uddin et al. (1994) and Swain and Dikshit (1997). Wen Xing et al. (1994) reported the beneficial effect of crossing carried out between genotypes having genetic distance $\left(\mathrm{D}^{2}\right)$ greater than 12.5. Thus it could be suggested that crosses should be made between genotypes belonging to the distant cluster for higher heterotic response.

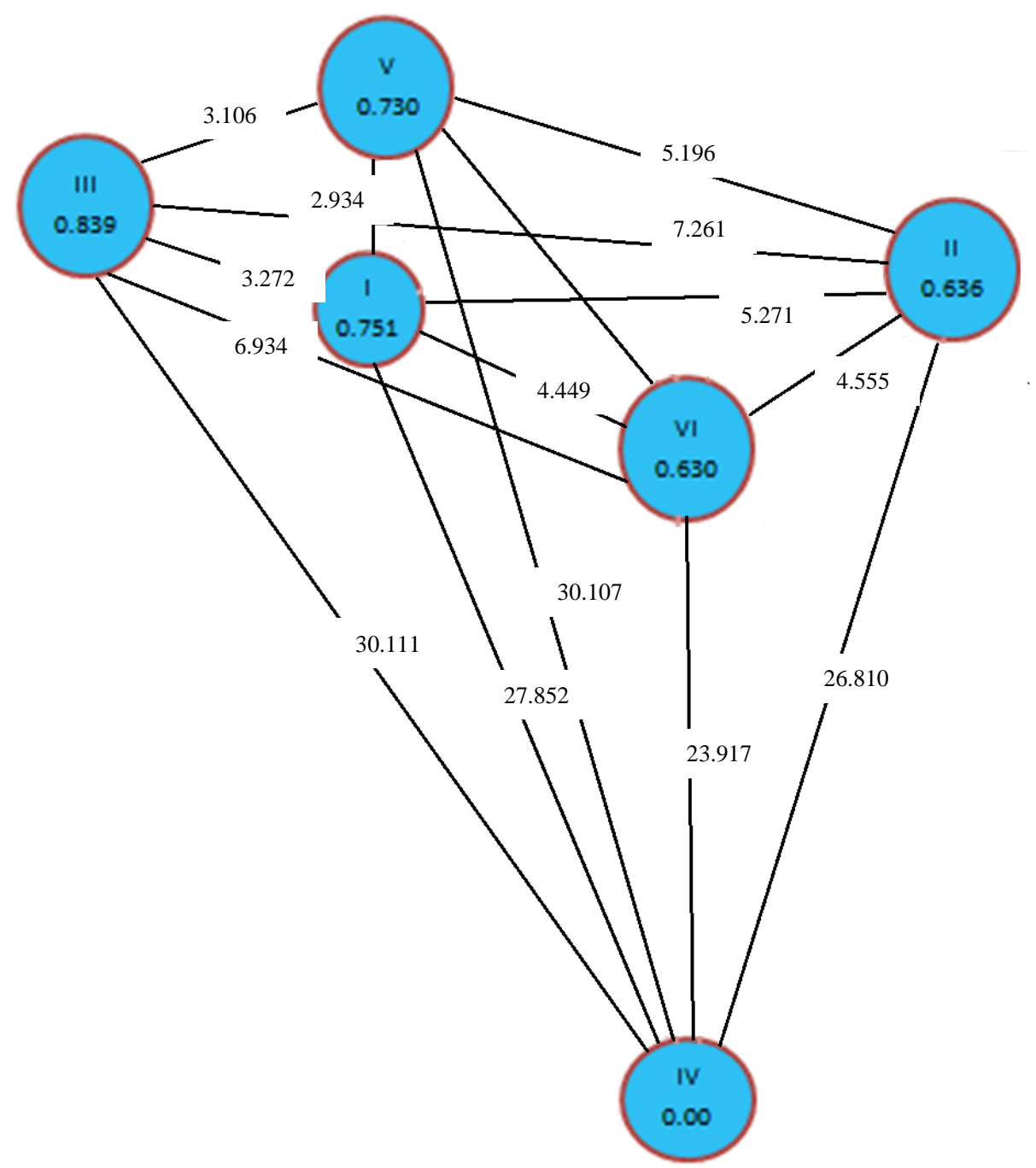

Fig. 2. Diagram showing the inter cluster distance and intra-cluster (inside the circle) distances of 64 muskmelon genotypes. 
The highest inter cluster distance between III and IV (30.111) suggesting that crossing between the desirable genotypes of the two clusters for getting greater heterotic effect. Moreover, the heterosis could also be exploited by crossing between genotypes with moderate diversity like I and IV, II and IV, IV and V and IV and VI (Fig.2). Szamosi et al. (2010) found heterosis by crossing between the genotypes with higher inter cluster distance.

\section{Non- hierarchical clustering}

With the application of covariance matrix for non-hierarchical clustering, the 64 genotypes were grouped into six different clusters. These results confirmed the clustering pattern of the genotypes according to their principal component analysis.

The cluster composition with different genotypes including their collection site is presented in Table 4 . On the basis of $\mathrm{D}^{2}$ analysis, the 64 genotypes were grouped into six clusters. The distribution pattern indicated that the maximum number of genotypes (20) included in cluster I followed by cluster V (12). Cluster III and VI had same (11) number of genotypes. The lowest number of genotypes was found in Cluster II. Diversity is generally associated with geographical diversity but genetic diversity is not directly related with geographic distribution (Luan et al, 2008 and Szamosi et al., 2010).

The clustering pattern of the genotypes under this study revealed that the genotypes originating from the same country did not form a single cluster. Even, the local genotypes collected from the same district did not form the same cluster which indicated that the geographic diversity is not always related to the genetic diversity and it might be due to the continuous exchange of germplasm among the researcher and grower. Therefore, it may be concluded that the selection of cultivars for hybridization should be based on genetic diversity instead of geographic diversity.

Table 4. Distribution and place of collection of 64 genotypes of muskmelon in different cluster

\begin{tabular}{c|c|ll}
\hline Cluster & Total no. & Genotype no. & Source of collection \\
\hline & BD2255 & Kushtia \\
& BD2262 & Kushtia \\
& BD2263 & Kushtia \\
& \multirow{2}{*}{20} & BD2264 & Kushtia \\
& BD2265 & Kushtia \\
& BD2266 & Munshigonj \\
& BD2268 & Kushtia \\
& BD2271 & Kushtia \\
& BD2279 & Faridpur
\end{tabular}


Table 4. Cont'd

\begin{tabular}{|c|c|c|c|}
\hline Cluster & Total no. & Genotype no. & Source of collection \\
\hline & & BD2282 & Faridpur \\
\hline & & BD2283 & Chittagong \\
\hline & & BD2285 & Jamalpur \\
\hline & & BD2289 & Mymensingh \\
\hline & & BD2292 & Tangail \\
\hline & & BD2293 & Tangail \\
\hline & & BD2295 & Tangail \\
\hline & & BD2301 & Comilla \\
\hline & & BD2305 & Sirajgonj \\
\hline & & BD2315 & Munshigonj \\
\hline & & BD2316 & Sirajgonj \\
\hline \multirow{9}{*}{ II } & \multirow{9}{*}{9} & BD2256 & Kushtia \\
\hline & & BD2258 & Munshigonj \\
\hline & & BD2273 & Kushtia \\
\hline & & BD2274 & Kushtia \\
\hline & & BD2286 & Jamalpur \\
\hline & & BD2290 & Tangail \\
\hline & & BD2300 & Comilla \\
\hline & & BD2306 & Sirajgonj \\
\hline & & BD2318 & Sirajgonj \\
\hline \multirow{11}{*}{ III } & \multirow{11}{*}{11} & BD2257 & Kushtia \\
\hline & & BD2267 & Kushtia \\
\hline & & BD2269 & Kushtia \\
\hline & & BD2275 & Kushtia \\
\hline & & BD2277 & Faridpur \\
\hline & & BD2278 & Faridpur \\
\hline & & BD2281 & Faridpur \\
\hline & & BD2297 & Tangail \\
\hline & & BD2298 & Comilla \\
\hline & & BD2304 & Sirajgonj \\
\hline & & BD2311 & Sirajgonj \\
\hline IV & 1 & BD2303 & Sirajgonj \\
\hline \multirow{7}{*}{$\mathrm{V}$} & \multirow{7}{*}{12} & BD2261 & Kushtia \\
\hline & & BD2272 & Kushtia \\
\hline & & BD2276 & Faridpur \\
\hline & & BD2280 & Faridpur \\
\hline & & BD2294 & Tangail \\
\hline & & BD2296 & Tangail \\
\hline & & BD2299 & Comilla \\
\hline
\end{tabular}


Table 4. Cont'd

\begin{tabular}{c|lll}
\hline Cluster & Total no. & Genotype no. & Source of collection \\
\hline & BD2307 & Sirajgonj \\
& BD2312 & Sirajgonj \\
& BD2313 & Sirajgonj \\
& BD2314 & Sirajgonj \\
& BD9160 & Pabna \\
& BD2259 & Kushtia \\
& BD2260 & Kushtia \\
& BD2287 & Mymensingh \\
& BD2288 & Mymensingh \\
& BD2291 & Tangail \\
& BD2302 & Sirajgonj \\
& BD2308 & Sirajgonj \\
& BD2309 & Sirajgonj \\
& BD2310 & Sirajgonj \\
& BD2317 & Sirajgonj \\
& BD2323 & Kushtia \\
\hline
\end{tabular}

Result of different multivariate techniques have been superimposed in Fig.1. The clustering pattern obtained with the apparent grouping pattern performed by nonhierarchical clustering.

\section{Cluster means for 12 characters in muskmelon}

Cluster means for twelve characters are presented in Table 5. Fruit length had the highest mean value in cluster VI $(16.8 \mathrm{~cm})$ followed by cluster IV $(16.4 \mathrm{~cm})$ and II $(16.2 \mathrm{~cm})$. The genotypes of cluster V $(15.1 \mathrm{~cm})$ produced the shortest fruit length. The highest fruit width was produced by the genotypes under the cluster IV $(8.3 \mathrm{~cm})$ followed by cluster II $(7.8 \mathrm{~cm})$, I $(7.5 \mathrm{~cm})$ and VI $(7.3 \mathrm{~cm})$. The lowest mean values of this trait were shown by cluster III $(6.9 \mathrm{~cm})$. Maximum fruit girth was found in Cluster IV $(26.2 \mathrm{~cm})$. Similar girth was found in Cluster II and VI $(25.5 \mathrm{~cm})$ and cluster III and V $(23.8 \mathrm{~cm})$ which had minimum girth among the clusters. Flesh thickness of muskmelon fruit was more or less equal $(1.5 \mathrm{~cm})$ in all clusters except cluster VI $(1.6 \mathrm{~cm})$ which has been marked as thicker groups compared to others. Cavity diameter was found highest in cluster IV $(5.2 \mathrm{~cm})$ and that the lowest cavity diameter was in Cluster III and VI (4.0 $\mathrm{cm})$. The heaviest fruit was found in cluster IV $(2533.3 \mathrm{~g})$. This cluster was composed of only one genotype (BD2303). Therefore, it may be revealed that genotype of this cluster could be used for developing high yielding variety of while the small size fruit was found in cluster V (372.5 g). Maximum number of fruits per plant (5.9) was found in cluster I and II. The highest number of seeds per fruit was found in cluster II (688) and that of the lowest number was in cluster III (249.5). Hundred seed weight was found maximum in cluster IV (2.1g) 
which was followed by cluster III and VI (1.9 g). The cluster II showed the highest seed yield $(74.7 \mathrm{~g})$ per plant and the cluster III showed the lowest seed yield (25.6g). The highest total fruit weight per plant was found in cluster IV $(13.5 \mathrm{~kg})$ which was far different from other clusters. The lowest total fruit weight was found in cluster III and V. Sweetness of fruit is indicated by brix \%. Cluster VI showed the highest brix \% (5.6). Hence, the genotypes of this cluster could be used for sweetened variety development. The lowest brix \% (5.0) was found in cluster IV: however it was less sweet.

Table 5. Cluster mean values for different characters of muskmelon genotypes

\begin{tabular}{l|c|c|c|c|c|c}
\hline Characters & I & II & III & IV & V & VI \\
\hline Fruit length (cm) & 15.8 & 16.2 & 15.6 & 16.4 & 15.1 & 16.8 \\
Fruit width (cm) & 7.5 & 7.8 & 6.9 & 8.3 & 7.1 & 7.3 \\
Fruit girth (cm) & 24.7 & 25.5 & 23.8 & 26.2 & 23.8 & 25.5 \\
Flesh thickness (cm) & 1.5 & 1.5 & 1.5 & 1.5 & 1.5 & 1.6 \\
Cavity diameter (cm) & 4.4 & 4.7 & 4 & 5.2 & 4.1 & 4 \\
Fruit size (g) & 557.3 & 606.3 & 399.1 & 2533.3 & 372.5 & 828.2 \\
Number of fruits / plant & 5.9 & 5.9 & 5.4 & 5.3 & 5.6 & 5.6 \\
Number of seeds / fruit & 334.4 & 688 & 249.5 & 297.3 & 456.8 & 429.7 \\
100 seed weight (g) & 1.6 & 1.8 & 1.9 & 2.1 & 1.7 & 1.9 \\
Seed yield / plant (g) & 31.8 & 74.7 & 25.6 & 33.7 & 43 & 44.3 \\
Total fruit weight / plant (kg) & 3.3 & 3.6 & 2.1 & 13.5 & 2.1 & 4.6 \\
Brix \% & 5.3 & 5.1 & 5.3 & 5 & 5.5 & 5.6 \\
\hline
\end{tabular}

Finally, based on multivariate analysis and application of covariance matrix for non-hierarchical clustering, 64 genotypes of muskmelon were grouped into six clusters indicating the existence of considerable diversity among the genotypes. Principal coordinate (PCA) analysis showed the highest inter genotypic distance (2.878) between the genotypes BD2303 and BD2313, while the lowest distance (0.249) was observed between genotypes BD2288 and BD2310. The most diverse genotypes were found in cluster III while the least diverse genotypes were found in Cluster VI. The inter cluster distances were larger than the intra cluster distances suggesting wider genetic diversity among the genotypes of different clusters. In addition, the inter-cluster distance was maximum between cluster III and IV (30.111) and the minimum distance was found between cluster I and V (2.934). Furthermore, mean values of various cluster pointed out the heavier fruit in cluster IV (2533.3g). So genotypes of this cluster could be used for developing high yielding variety. Sweetness of fruit is indicated by brix reading. Cluster VI showed the highest brix reading. Hence, the genotypes of this cluster could be used for sweetened variety development of muskmelon. 
Hybridization between the genotypes of cluster IV and those of cluster VI could develop high yielding sweet muskmelon variety(s).

\section{Conclusion}

Genetic diversity of the muskmelon was assessed using multivariate technique. A considerable range of genetic diversity was found in the genotypes. The cluster analysis grouped the genotypes into six divergent clusters. The highest genotypes possessed in Cluster I. The Principal component analysis revealed that the first principal axis largely accounted for the variation among the genotypes which alone contributed $25.65 \%$ of the variations. The maximum fruit weight per plant was found in cluster IV and the genotypes of this cluster could be used for developing high yielding variety. TSS indicates the sweetness of fruits. The genotype BD2312 ( $8^{0}$ brix) was found as the sweetest muskmelon among the tested genotypes.

\section{References}

Baenziger, P. S., W. K. Russell, G. L. Graef, and B. T. Campbell. 2006. Improving lives. Crop sci. 46(5): 2230-2244.

Das, P.K. and T.D. Gupta. 1984. Multivariate analysis in Blackgram. Indian J. Genet., 44(2): 243-247.

Digby, P., N. Galway and P. Lane. 1989. GENSTAT 5: A Second Course. Oxford Science Publications, Oxford. Pp.103-108.

Escribano, S. and A. Lázaro. 2009. Agro-morphological diversity of Spanish traditional melons (Cucumis melo.) of the Madrid provenance. Genet. Resour. Crop Evol. 56:481-497.

Flowers, T. J. 2004. Improving crop salt tolerance. J. Exp. Bot. 55 (396) : 307-319.

Hancock, J. F. 2012. Plant evolution and the origin of crop species. CABI. Pp. 492-500.

Henane, I., N. Mezghani, I. Tlili, R.Thouraya, G. Ismail, A. Kamel and J. Hager. 2013. Agro-morphological characterization and assessment of variability in local germoplasm of $C$. melo in Tunisia. Journal of Biodiversity and Environmental Sciences (JBES). 3(12): 198-207.

Jeger, M.I., D. Garethojones and E. Griffiths. 1983. Genetic divergence in wheat. Indian J. Genet. 43: 63-67.

Kalloo, J. Dixit and A.S. Sidhu. 1982. Genetic divergence in muskmelon (Cucumis melo L.). Genet. Agr. 36: 1-8.

Luan, F.S., I. Delannay and J.E. Staub. 2008. Chinese melon (C. melo) diversity analyses provide strategies for germplasm curation, genetic improvement and evidentiary support of domestication patterns. Euphytica 164 (2): 445-461.

Murty, B.R. and V. Arunachalam. 1966. The nature of genetic divergence in relation to breeding system in crop plants. Indian J. Genet. 26A: 188-198. 
Nasrabadi, H.N., H. Nemati, A. Sobhani and M. Sharifi. 2012. Study on morphologic variation of different Iranian melon cultivars (Cucumis melo). African J. Agric. Res. 7(18): 2764-2769.

Saxena, K. B. 2005. Pigeonpea Cajanus cajan (L.) Millsp. Genetic resources, chromosome engineering, and crop improvement series. Grain legumes. 1: 85-115.

Singh. R.K. and B.D. Chaudhary. 1985. Biomentical Methods in Quantitative Genetic analysis. Kalayani publishers, New Delhi, India. P. 318.

Stuthman, D. D., K. J. Leonard, and J. Miller-Garvin. 2007. Breeding crops for durable resistance to disease. Advances in Agron. 95: 319-367.

Swain, D. and U.N. Dikshit. 1997. Genetic divergence in rabi sesame. Indian J. Genet. 57: 296-300.

Swaminathan, M.S. 1983. Genetic conservation: microb to man. Presidential Address XV. International Congress of Genetics, New Delhi. P. 31.

Szamosi, C., I. Solmaz, N. Sari and C. Barsony. 2010. Morphological evaluation and comparison of Hungarian and Turkish melon (C. melo) germplasm. HortScience. 124: $170-182$.

Tomoka, N. 1991. Genetic diversity and land race differentiation of mung bean (Vigna radiata (1) Wilczek) and evaluation of its wild relatives (The sub-genus ceratotropics) as breeding materials. Tech. Bull. Trop. Res. Centre, Japan, No. 28. Ministry of Agri. Forestry and Fisheries, Japan. P.1.

Trimech, R., Y. Zaouali and M. Boussaid. 2013. Genetic variation in Tunisian melon ( Cucumis melo L.) germplasm as assessed by morphological traits. Genet. Resour. Crop Evol. Springer Science. 10: 1007.

Uddin, M.N., F.W. Ellison, L. O’brien and B.D.H. Latter. 1994. Relationships among attributes of pure lines of bread wheat derived from heterotic $\mathrm{F}_{1}$ hybrids. Aust. J. Agric. Res. 45: 601-611.

Wen Xing, W., Z. Hong, L.F. Yin, W. Shuangling, W.X. Wei, H. Zhang, F.Y. Lu and S.L. Wei. 1994. Principal components analysis and genetic distance estimation and their application in sesame breeding programme. Acta Agric. Boreali Sinica. 9(3): 29-33.

Zhang, C., A.S. Pratap, N. Senthil, S. Atarajan, T. Koba, L. Pugalendhi, S. Kikuchi and H. Sassa. 2012. Evaluation of morphological and molecular diversity among South Asian germplasm of Cucumis sativus and Cucumis melo. International Scholarly Research Network. ISRN Agronomy. 2012: P. 11. 